\title{
$X$-Parameter Measurement of Pulse-Compression Nonlinear Transmission Lines
}

\author{
Ming Li, Rony E. Amaya, Robert G. Harrison, and N. Garry Tarr \\ Department of Electronics, Carleton University, Ottawa, Ontario, Canada K1S 5B6 \\ Correspondence should be addressed to Ming Li, mli@doe.carleton.ca
}

Received 19 July 2009; Accepted 30 September 2009

Academic Editor: Muhammad Taher Abuelma'atti

Copyright () 2010 Ming Li et al. This is an open access article distributed under the Creative Commons Attribution License, which permits unrestricted use, distribution, and reproduction in any medium, provided the original work is properly cited.

$X$-parameters provide a powerful and eminently practical solution for interoperable measurement, modeling, and simulation of nonlinear microwave and RF components. Using $X$-parameters to do large-signal measurements has been brought into the spotlight. This paper introduces a new $X$-parameter application: measuring large-signal behaviour of pulse-compression nonlinear transmission lines (NLTLs). A specially configured Nonlinear Vector Network Analyzer (NVNA) was used to measure the $X$ parameters in the frequency-domain, and then the measured data was transformed into a nonlinear time-domain waveform. The results show both rise-time and fall-time reduction (double-edge compression) as expected and indicate that this newly developed $X$-parameter method implemented with an NVNA could correctly predict NLTLs' pulse-compression performance.

\section{Introduction}

All active devices exhibit nonlinear behaviour to some degree. In general, the higher the operation frequencies, the higher the degree of nonlinear behaviour. The rapidly developing semiconductor chip manufacturing industry has brought RFIC designs into a much higher frequency level over the past decade. This makes large-signal nonlinear behavior modeling and measurements more critical. Unfortunately, making nonlinear measurements is not an easy task. Most currently available tools and models for accomplishing this goal are generally difficult to use and do not provide all the information required. Most importantly, large-signal nonlinear calibration is too complicated to be accomplished, resulting in an inability to accurately measure the nonlinear effects of active RF devices.

A few years ago, Root and Wood at Agilent Technologies Inc. developed the $X$-parameter concept [1]. Mathematically, $X$-parameters are an extension of $S$-parameters [2] to largesignal conditions. They can be used to represent and analyze the nonlinear behavior of RF components in a much more robust and complete manner. $X$-parameters include both harmonics and intermodulation products, corresponding to the nonlinear spectral components. More details on $X$ parameter theory are given in Section 2.
$X$-parameters of a nonlinear device can be measured by using the new Agilent Nonlinear Vector Network Analyzer (NVNA). There are two primary methods used in measuring the nonlinearity of a device under test (DUT), the first being nonlinear component characterization, the second being $X$ parameter methodology. The first one provides calibrated, vector-corrected waveforms of the incident, transmitted and reflected waves from the DUT. With the use of a poly-harmonic phase reference, vector and power calibration, including spectrum amplitude and cross-frequency phase calibration, can be done. This makes possible the removal of the systematic error terms. With the second method, all receivers must be measured simultaneously for each frequency point. The measured $X$-parameters can be characterized and extracted by the Agilent design software ADS2009 [3]. The "PHD (Poly-Harmonic Distortion) block" component in the software converts the measured data file into a device-specific instance. The measured results can then be converted into time-domain waveforms or used for further simulation and design. The related work has been published in [4-6]. A simplified representation of the NVNA and its interoperability with ADS is shown in Figure 1.

In this paper, Section 2 presents the $X$-parameter principle. Section 3 describes the NLTL pulse compression. The NLTL was designed, simulated, and fabricated on a 


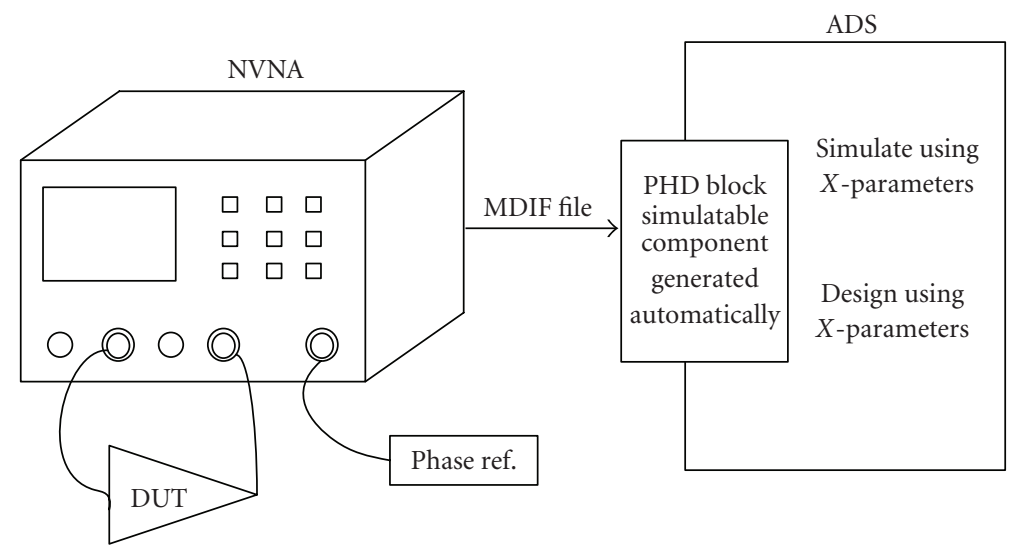

FIGURE 1: Simplified configuration of an NVNA and ADS workbench for nonlinearity measurements.

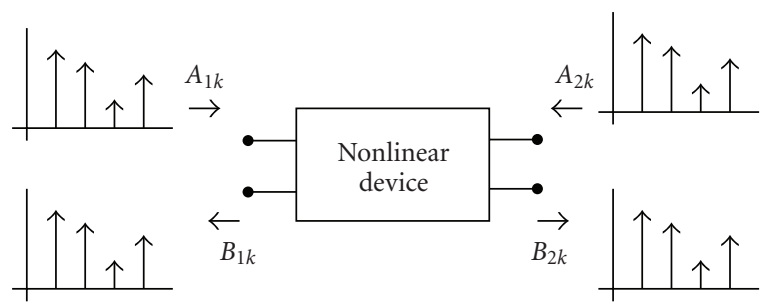

FIGURE 2: Scattering wave transmission.

commercial CMOS $0.18-\mu \mathrm{m}$ chip $[7,8]$. By using the NVNA and $X$-parameters, we are able to measure the NLTL largesignal nonlinear behavior. The measured results are given in Section 4. The tested results are as expected and have good agreement with the simulated results. This work proves that $X$-parameters are suitable to precisely predict largesignal nonlinear behavior of pulse-compression NLTLs. The application can also be easily extended to other NLTL measurements.

\section{X-Parameter Principle}

$X$-parameters were first described (under another name) as early as 2005 [1]. Assume that $A$ represents an incident wave and that $B$ represents the scattered or reflected wave in Figure 2 [1]. For the incident and reflected wave quantities, $A_{1 k}, A_{2 k}, B_{1 k}$, and $B_{2 k}$, the first subscript refers to the signal port and the second to the harmonic index. A harmonic index of 1 represents the fundamental component, a harmonic index of 2 refers to the second harmonic, and so on. Using a generalized frequency-domain black-box modeling technique, the output spectral components, $B_{1 k}$, and $B_{2 k}$, correlate with the input spectral components, $A_{1 k}$ and $A_{2 k}$, and can be represented by

$$
\begin{aligned}
& B_{1 k}=F_{1 k}\left(A_{11}, A_{12}, \ldots, A_{21}, A_{22}, \ldots\right), \\
& B_{2 k}=F_{2 k}\left(A_{11}, A_{12}, \ldots, A_{21}, A_{22}, \ldots\right),
\end{aligned}
$$

where the letter $F$ denotes a correlation function.

Two important techniques used in $X$-parameter theory.
2.1. Phase Normalization. Phase-normalized quantities are used to simplify the mathematics. $A_{11}$ is used as the phasereference component. Since $A_{11}$ is the dominant largesignal input component, all other input components (at the harmonic frequencies) are relatively small compared to $A_{11}$. First, a component $P$ is defined which has a phase equal to that of $A_{11}$, and unit amplitude, as shown in (2):

$$
P=e^{j p\left(A_{11}\right)},
$$

where $p\left(A_{11}\right)$ represents the phase of $A_{11}$, and $j=\sqrt{-1}$. The normalized quantities, denoted by the superscript $N$, are then calculated by

$$
A_{m k}^{N}=A_{m k} P^{-k}, \quad B_{m k}^{N}=B_{m k} P^{-k},
$$

where $k$ refers to the harmonic index of that component. Note that $A_{11}^{N}=\left|A_{11}\right|$ is a positive real number.

2.2. Harmonic Superposition. In general, the superposition principle and linear relationships are inapplicable to largesignal nonlinear systems. However, in many practical cases there is only one dominant large-signal input component $\left(A_{11}\right)$, so that it is valid to use the superposition principle for the relatively small phase-normalized input components. This is called the "harmonic superposition" principle [1].

Considering only $A_{1}$ and $B_{2}$ in Figure 2, nonzero $A_{11}$ will contribute a significant harmonic to $B_{2}$. Suppose that a relatively small harmonic component $A_{12}$ is present at the input. This will result in a change in the output spectrum $B_{2}$ at the second harmonic. The same principle holds for higher harmonics. The overall deviation of the output spectrum $B_{2}$ is the superposition of all the individual deviations. The $X$ parameters are given by (4) [1]:

$$
B_{m k}^{N}=\sum_{n k} X_{m k n k}\left(A_{11}^{N}\right) A_{n k}^{N}+X_{m k n k}^{\prime}\left(A_{11}^{N}\right) A_{n k}^{N^{*}} .
$$

The phase-normalized $B^{N}$ waves are the output quantities. They are written as a linear combination of the input phasenormalized $A^{N}$ quantities and their conjugates (denoted by the superscript*). Each $X$-function is defined by four 


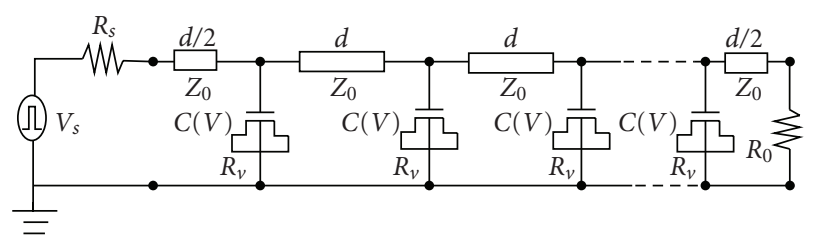

FIgURe 3: Circuit diagram of the NLTL configuration.

subscripts: the first two denote the port and harmonic index of the associated output component $B$, and the latter two describe the port and harmonic index of the associated input component $A$.

$X$-parameters have some unique features compared to $S$-parameters. In particular, $X$-parameters are nonlinear functions of the input signal power and can map incident signals at specific frequencies to other frequencies. Since $X$-parameters include many cross-frequency dependencies, there are more $X$-parameters than $S$-parameters.

\section{Pulse-Compression NLTL Circuits}

The NLTLs discussed here consist of coplanar waveguide (CPW) transmission lines interleaved with NMOS varactors (see Figure 3). $V_{s}$ and $R_{s}$ characterize the pulse source, $R_{0}$ is the load impedance, $Z_{0}$ represents the characteristic impedance of the CPW, $d$ is the section length of the CPW, while $C(V)$ and $R_{v}$ represent, respectively, the nonlinear capacitance and the series loss resistance of each NMOS varactor.

An NLTL has three fundamental and significant characteristics: nonlinearity, dispersion, and dissipation. These define the NLTL's functions and behavior. Nonlinearity is due to the voltage-dependent varactor capacitance, dispersion to the periodicity of the NLTL, and dissipation to the finite conductivity of the CPW conductors and the series resistance $R_{v}$ of each varactor.

NLTLs can function as pulse-compression components [9]. Two cutoff frequencies are used to describe this property, the varactor cutoff frequency $f_{c}$ and the Bragg frequency $f_{B}$ :

$$
f_{c}=\frac{1}{2 \pi R_{V} C(V)}, \quad f_{B}=\frac{1}{\pi d \sqrt{L\left(C_{0}+C(V) / d\right)}},
$$

where $L$ and $C_{0}$ are the per-unit-length inductance and capacitance of the CPW, and $d$ is the section length of the NLTL. When $f_{c} / f_{B}$ is $4 \sim 6$, the NLTL is in a weak dispersion mode and can produce shock waves. In other words, if we input a pulse wave, the rise or fall edge of the pulse wave at the output will be sharpened.

Nonlinearity of the MOS varactors, together with the transmission line parameters, determines the nature of the nonlinear wave propagation along the NLTL. Varactors used in pulse-compression NLTLs require a large cutoff frequency $f_{c}$ for high-order harmonic generation and a $C(V)$ characteristic optimized for a high degree of nonlinearity. Specific varactor structures can provide either monotonic or nonmonotonic $C(V)$ characteristics. As pointed out in [8], a monotonic $C(V)$ is beneficial for single-edge pulse

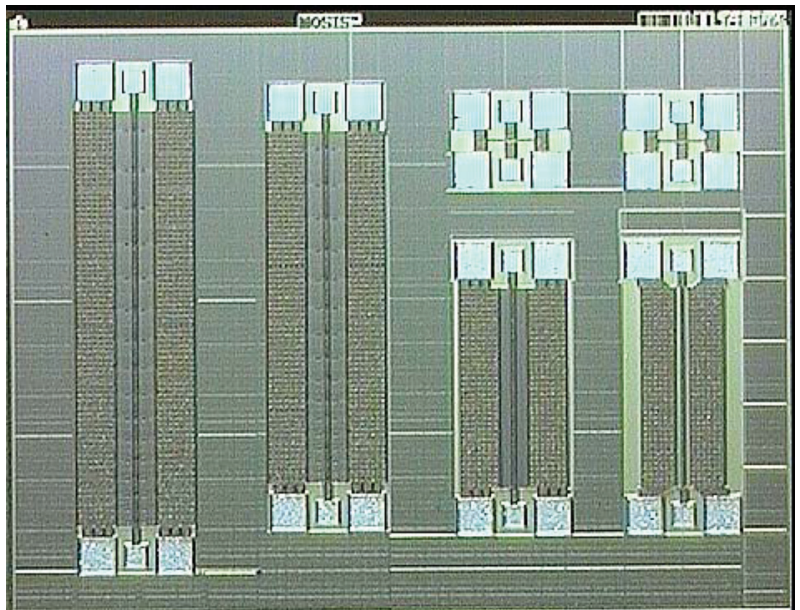

FIgURe 4: Pulse-compression NLTL chip.

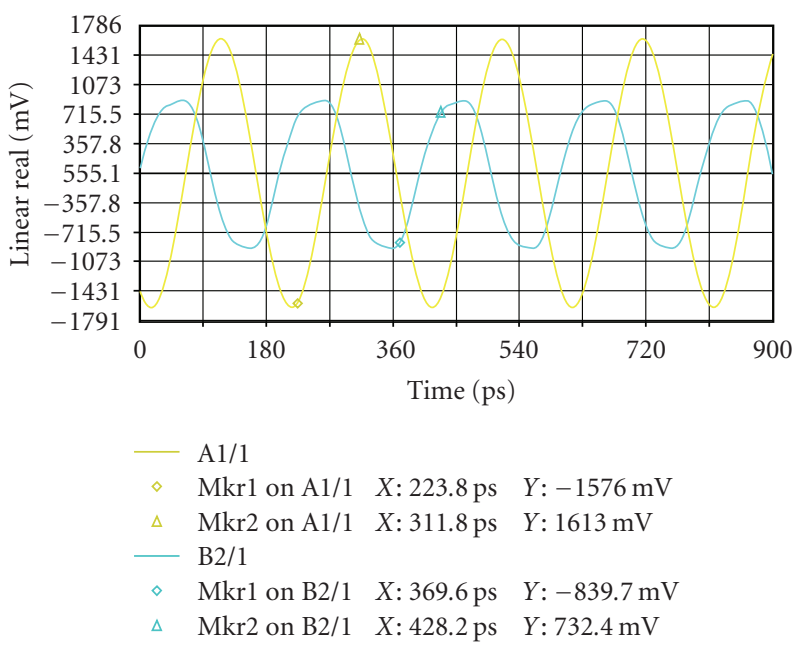

FIgURE 5: Measured NLTL time-domain waveforms for a $13.9 \mathrm{dBm}$ input at $5 \mathrm{GHz}$.

compression while a nonmonotonic $C(V)$ is more suited for double-edge compression.

We realized NLTL pulse-compression circuits on a $1.5 \times$ $2.0 \mathrm{~mm}^{2}$ silicon chip, as shown in Figure 4. The left-most line in Figure 4 is a double-edge pulse-compression NLTL. It has 16 sections and a total length of $1.06 \mathrm{~mm}$. In this design both slow-wave-CPW and tapered-line technologies are used [7].

\section{NLTL Nonlinearity Measurement}

The nonlinearity measurements were done at the microwave laboratory of the Communications Research Centre (CRC), Ottawa, Canada. An N5242A-type NVNA was provided by Agilent Technologies on a "demonstrator" basis. The testing setup is shown in Figure 1. The maximum frequency capability of the N5242A system at the time was $26 \mathrm{GHz}$. The input signal we chose was a $5-\mathrm{GHz}$ sinusoidal wave since the pulse input (or square-wave input) feature was still under development by the company. This means that at the output 


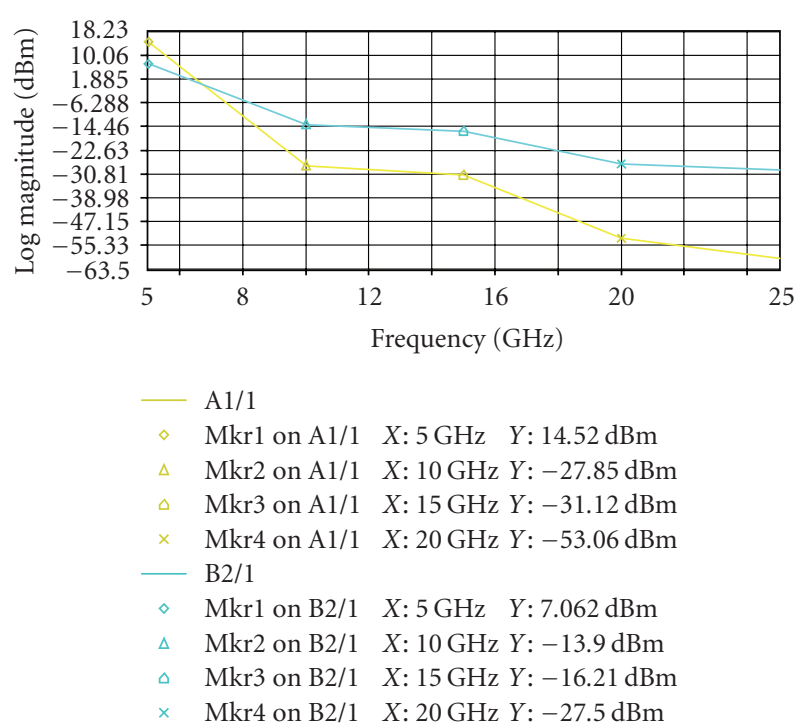

Figure 6: Measured frequency-domain NLTL spectrum for a $13.9 \mathrm{dBm}$ input at $5 \mathrm{GHz}$.

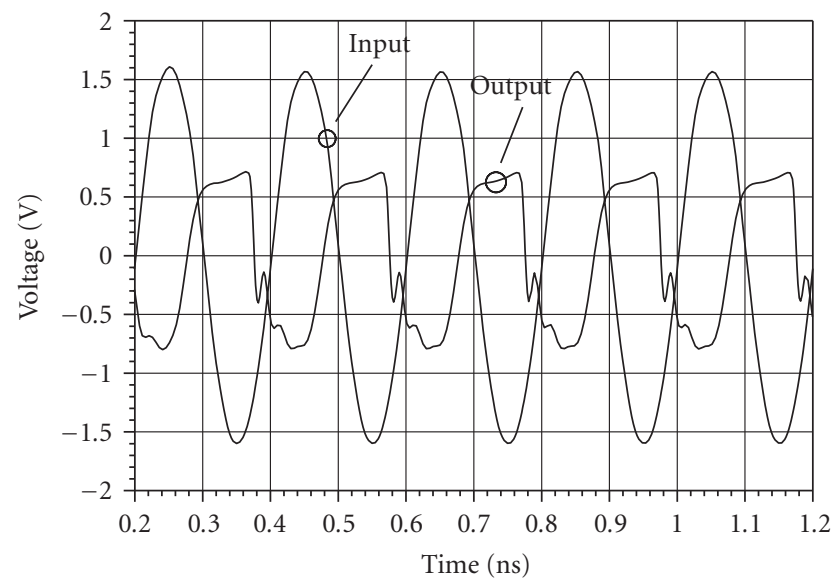

FIGURE 7: The simulated NLTL result with a $5 \mathrm{GHz}, 1.6 \mathrm{~V}$-amplitude input.

of an NLTL, the highest harmonic that could be measured, at $25 \mathrm{GHz}$, was the 5 th. Figure 5 shows time-domain results for the double-edge compression NLTL with an input at $13.9 \mathrm{dBm}$, which gives an input with $1.6 \mathrm{~V}$-amplitude. Figure 6 gives frequency-domain results for the same device. In Figures 5 and 6, A1 (yellow) indicates the input, B2 (blue), the output voltage waves. In Figure 6, markers Mkr1, Mkr2, $\mathrm{Mkr} 3$, and so forth identify the amplitudes of the 2nd, 3rd, 4 th, and so fifth harmonics.

The time-domain result shows strong double-edge compression with the output B2 is squarer than the input. The frequency-domain result shows that more energy is accumulated in the higher harmonic components. Compared to the input harmonics, the second output harmonic increases by $14 \mathrm{dBm}$, and the third one increases by $16 \mathrm{dBm}$.

To validate the testing results, the simulation was done in ADS. Figure 7 shows the results. Since the highest harmonic

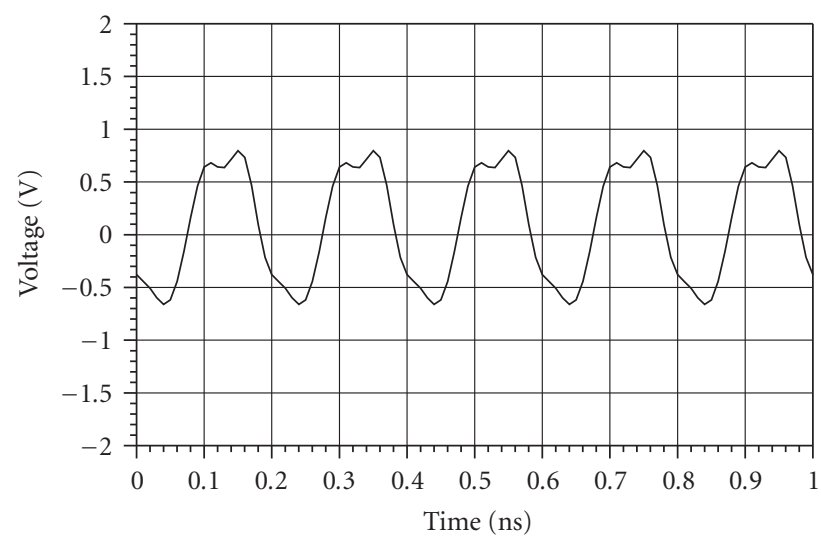

FIGURE 8: Reconstructed waveform using only 5 lowest output harmonics.

the NVNA can cover is the 5th, we reconstructed the output waveform using only the 5 lowest output harmonics. Figure 8 shows the result. It has a good agreement with Figure 5.

\section{Conclusion}

We previously showed that silicon NLTLs can be used in pulse-compression circuitry $[7,8]$. However, our work then was restricted to simulation and small-signal on-chip testing because of the lack of proper methodology and equipment for the large-signal nonlinearity measurements. The $X$-parameter method and NVNA described in this paper allowed us to do that. The good agreement between the measured and simulated output waveforms proves that silicon pulse-compression NLTLs can be successful. Our work also shows that the new testing technology is a good candidate for large-signal nonlinearity measurement in the RFIC industry.

\section{References}

[1] J. Wood and D. E. Root, Fundamentals of Nonlinear Behavior Modeling for RF and Microwave Circuits, chapter 5, Artech House, Boston, Mass, USA, 2005.

[2] D. M. Pozar, Microwave Engineering, chapter 5, AddisonWesley, Reading Mass, USA, 1990.

[3] http://www.home.agilent.com/agilent/product.jspx?nid=-34 360.0.00\&cc $=$ US\&lc $=$ eng.

[4] D. E. Root, J. Verspecht, D. Sharrit, J. Wood, and A. Cognata, "Broad-band poly-harmonic distortion (PHD) behavioral models from fast automated simulations and large-signal vectorial network measurements," IEEE Transactions on Microwave Theory and Techniques, vol. 53, no. 11, pp. 3656-3664, 2005.

[5] J. Verspecht, D. Gunyan, J. Horn, X. Jianjun, A. Cognata, and D. E. Root, "Multi-tone, multi-port, and dynamic memory enhancements to PHD nonlinear behavioral models from largesignal measurements and simulations," in Proceedings of the IEEE MTT-S International Microwave Symposium Digest (IMS '07), pp. 969-972, Honolulu, Hawaii, USA, June 2007.

[6] J. Horn, D. Gunyan, L. Betts, C. Gillease, J. Verspecht, and D. E. Root, "Measurement-based large-signal simulation of 
active components from automated nonlinear vector network analyzer data via X-parameters," in Proceedings of the IEEE International Conference on Microwaves, Communications, Antennas and Electronic Systems (COMCAS '08), pp. 1-6, TelAviv, Israel, May 2008.

[7] M. Li, R. E. Amaya, J.-M. Duchamp, P. Ferrari, R. G. Harrison, and N. G. Tarr, "Low-loss low-cost all-silicon CMOS NLTLs for pulse compression," in Proceedings of the IEEE MTT-S International Microwave Symposium Digest (IMS '07), pp. 449452, Honolulu, Hawaii, USA, June 2007.

[8] L. Ming, R. G. Harrison, R. E. Amaya, J.-M. Duchamp, P. Ferrari, and N. G. Tarr, "CMOS varactors in NLTL pulsecompression applications," in Proceedings of the 37th European Microwave Conference (EUMC '07), pp. 1405-1408, Munich, Germany, October 2007.

[9] M. J. W. Rodwell, S. T. Allen, R. Y. Yu, et al., "Active and nonlinear wave propagation devices in ultrafast electronics and optoelectronics," Proceedings of the IEEE, vol. 82, no. 7, pp. 1037-1059, 1994. 

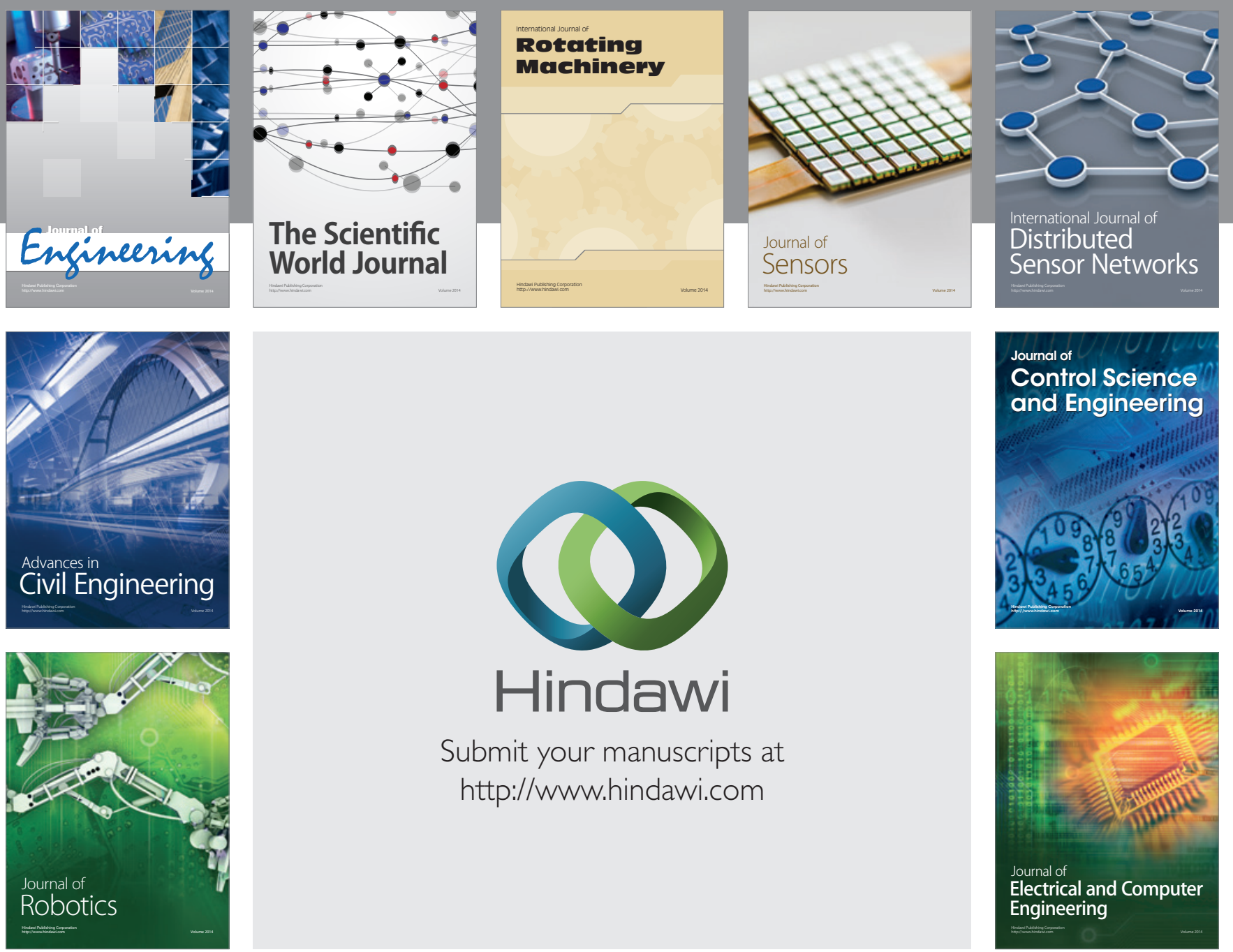

Submit your manuscripts at

http://www.hindawi.com
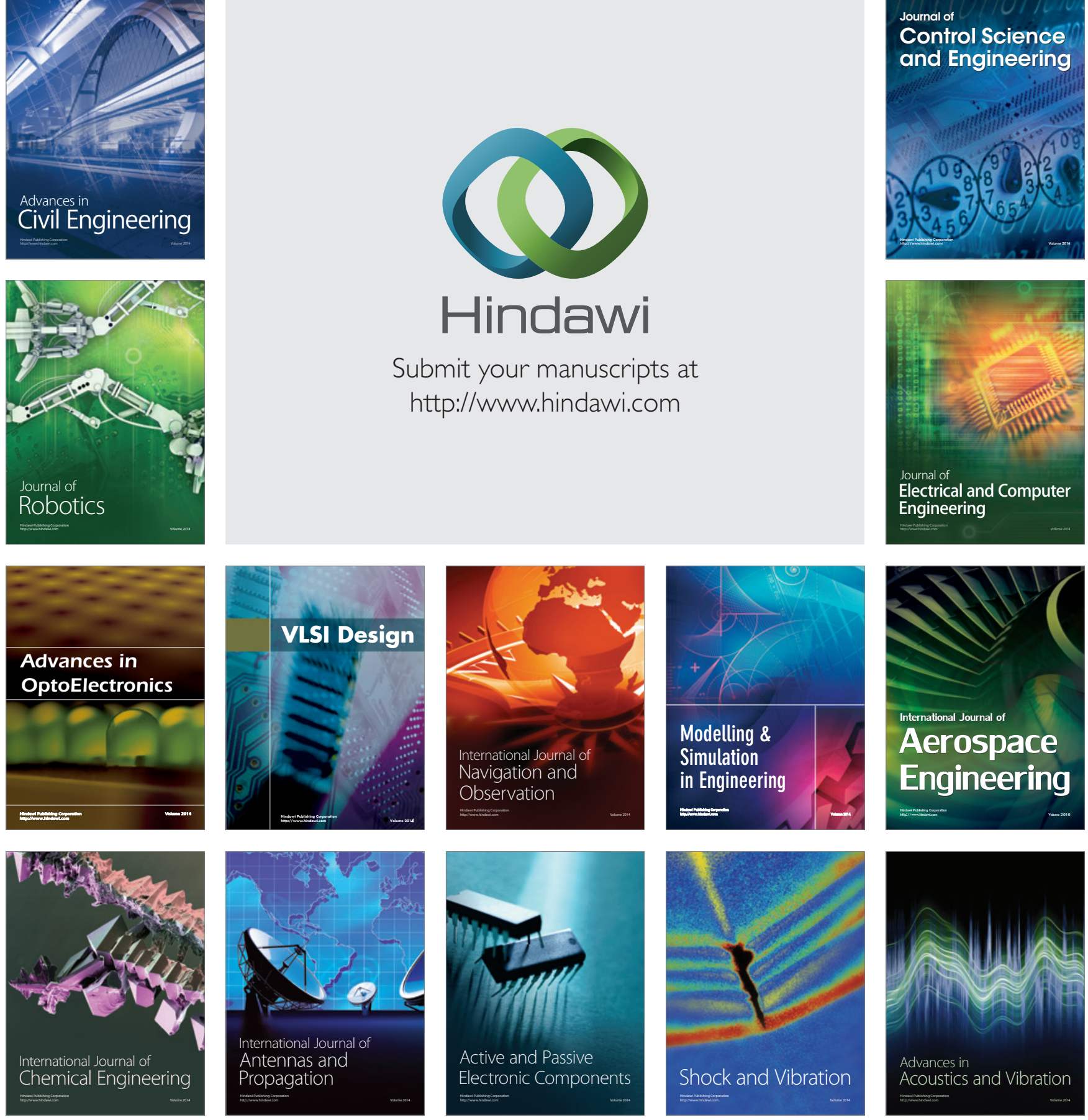